\title{
Intake of a QHCl-sucrose solution by carbohydrate-deprived rats
}

CHARLES KEMMLER ${ }^{1}$ AND JOHN EXNER, JR. BOWLING GREEN STATE UNIVERSITY

Carbohydrate-deprived rats were given an ad-lib choice between an aversively masked sucrose solution and water. Without an opportunity to associate the aversive taste with a beneficial post-ingestion effect, peripheral palatability inhibited selection in accordance with need. With this experience, however, aversive taste was bypassed in favor of selection according to dietary need.

Palatability, need, and previous experience affect food intake (Young, 1966). Need-free rats, for example, did not prefer any quinine hydrochloride (QHCl) solution to water (Young et al, 1963). Similarly, adding QHCl to either water (Corbit, 1965) or food (Wright, 1965) yielded decreased intake. However, early exposure to QHCl (Warren \& Pfaffmann, 1959) or general food deprivation (Tenen \& Miller, 1964) increased toleration of it when added to food or milk. Jacobs (1962) has indicated that ad lib choice situations between sugars and saccharin solutions are a function of need state, e.g., satiation, hunger, or thirst.

The present study examined QHCl toleration under a specific carbohydrate deprivation using a Low Carbohydrate Diet (LCD). ${ }^{2}$ Prior work indicated that an adulterated diet would not be ingested in sufficient quantity to equal caloric intake of standard chow (Janowitz \& Grossman, 1949; Smith et al, 1962). The deficiency would have to be made up through intake of the aversively masked sucrose solution. The aforementioned studies involving QHCl employed a one choice situation. The present investigation had a two choice ad lib format. There was also a one choice situation followed by the two choice condition to establish whether experience with a needed solution would increase its subsequent ingestion.

Method

Thirty-six 40-day-old male hooded rats were individually housed with two graduated cylinders and a food cup. All solutions were prepared with distilled water ${ }^{3}$, and chow pellets were ground to the consistency of the LCD. Animals were placed on ad lib schedules, and daily recordings were obtained for the following: body weight, chow or LCD intake, water intake, and QHCl-sucrose solution intake.

One pilot study was conducted to establish that LCD was insufficient as the sole source of calories. Criterion was comparative weight gain for an eightday period by $10 \mathrm{Ss}$ receiving LCD and water, and 10 Ss receiving chow and water. In addition, a second pilot study determined the aversion level of a $10 \mathrm{gm} /$ $100 \mathrm{ml}$ sucrose solution as a function of adding different quantities of QHCl. Aversion was defined as having less than $15 \%$ of total daily liquid intake from the QHC1-sucrose solution bottle, and at least $85 \%$ of intake from the water bottle. Six $\mathrm{Ss}$ were offered water, chow, and one of six different QHCl-sucrose solutions each day for six days. One solution was used in the experiment.

The experiment proper consisted of three consecutive phases of eight, six, and eight days duration. During the three phases 10 Ss (LCD group) were on ad lib schedule of LCD and QHCl-sucrose solution. Another group of $10 \mathrm{Ss}$ (chow group) had chow and the solution during the three phases.

In addition, water was available to both groups during phase 1 and phase 3 . The conditions were identical during phases 1 and 3 except for the temporal positioning of phase 2 before phase 3 . Because no water bottle was available during phase 2, reduction of thirst was possible only through intake of the QHCl-sucrose solution. Therefore, while both groups needed the solution for a source of water, the LCD group also needed the solution for its source of carbohydrate-sucrose.

Resulis and Discussion

In the one pilot study, chow rats exhibited a significantly greater weight gain than the LCD rats $(t=4.83, p<.01)$. In the other pilot study $16 \mathrm{mg}$ of QHCl added to the sucrose solution best satisfied the criterion of aversion and was therefore used in the experiment proper.

Figure 1 indicates that the LCD group gained sig-

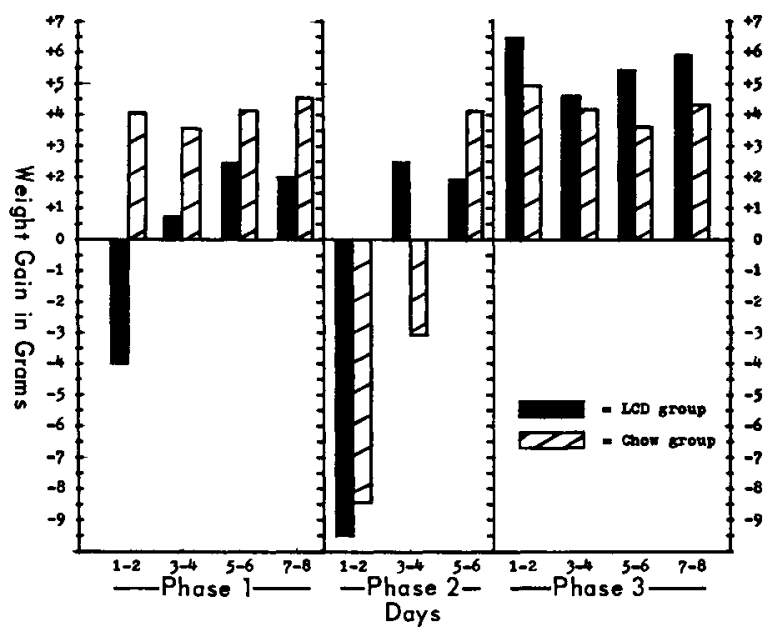

Fig. 1. The two-day sum weight gains by LCD rats and Chow rats during the three consecutive phases of the experiment proper. 


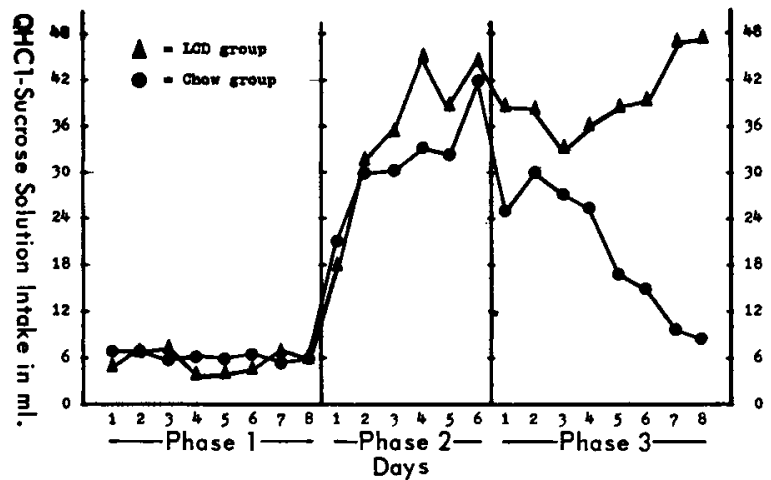

Fig. 2. The ingestion of QHC1-sucrose solution by LCD rats and Chow rats during the three consecutive phases of the experiment proper.

nificantly less weight than the chow group during phase $1(t=4.04, p<.01)$, whereas they gained just as much as the chow group during phase 3 . Difference t-tests indicated that these results were not a function of either the water intake or the food (LCD or chow) intake. However, Fig. 2 illustrates that the weight gain results were related to the $\mathrm{QHCl}-$ sucrose solution intake. While there was no difference in solution intake between the groups during phase 1 , the LCD group did ingest significantly more of the solution during phase 3 than the chow group $(F=4.58, p<.05$ ).

Because of the solution's aversive taste, only minimal intake occurred in the two bottle choice during phase 1 . As a result the LCD group had no opportunity to associate the $\mathrm{QHCl}$ taste cue with need reduction. In phase 3, however, the LCD group did ingest according to need by drinking "enough" QHCl-sucrose solution. The difference between phase 1 and phase 3 intake appears to be a function of phase 2 . Under conditions of thirst in phase 2, both groups ingested about six times more solution than they had on any day during phase 1 .

In phase 3 the correct choice of QHCl-sucrose solution in accordance with the specific need for carbohydrate by the LCD group was because of, and in spite of, the aversive QHCl taste cue. The LCD group had developed a learned appetite for the taste of QHCl. This occurred only after a significant solution intake during phase 2, which allowed opportunity to experience a beneficial post-ingestion effect with the aversive taste. As long as this post-ingestion experience can take place, even an aversively masked food will be ingested in accordance with a specific dietary need.

\section{References}

Corbit, J. D. Hyperphagic hyperactivity to adulteration of drinking water with quinine HC1. J. comp. physiol. Psychol., 1965, 60, 123-124.

Jacobs, H. L. Some physical, metabolic, and sensory components in the appetite for glucose. Amer. J. Physiol., 1962, 203, 10431054.

Janowitz, H. D., \& Grossman, M. I. Effects of variations in nutritive density on intake of food in dogs and rats. Amer. J. Physiol., $1949,158,184-193$.

Smith, M., Pool, R., \& Weinberg, H. The role of bulk in the control of eating.J. comp, physiol. Psychol., 1962, 55, 115-120.

Tenen, S. S., \& Miller, N. Strength of electrical stimulation of lateral hypothalamus, food deprivation, and tolerance for quinine in milk. J. comp. physiol. Psychol., 1964, 58, 55-62.

Warren, R., \& Pfaftmann, C. Early experience and taste aversion. J. comp. physiol. Psychol., 1959, 52, 263-266.

Wright, J. H. Modifications in rats diurnal activity patterns as function of opportunity for reinforcement by ingestion. J. comp. physiol. Psychol., 1965, 59, 463-465.

Young, P. T. Hedonic organization and regulation of behavior. Psychol. Rev., 1966, 73, 59-86.

Young, P. T., Burright, R. G., \& Tromater, L. Preferences of the white rat for solutions of sucrose and quinine hydrochloride. Amer. J. Psychol., 1963, 76, 205-217.

\section{Notes}

1. Now at Louisiana State University, Baton Rouge, Louisiana. 2. Purchased from Nutritional Biochemicals Corporation, Cleveland, Ohio.

3. The QHC 1 was donated by S. B. Penick \& Company, New York, New York. 